\title{
LA PRESENTAZIONE DELLE (IR)REGOLARITÀ FLESSIVE NELLE GRAMMATICHE D'ITALIANO PER STRANIERI: IL CASO DEI TEMPI PASSATI DELL'INDICATIVO
}

Abstract: Tra le questioni grammaticali più difficoltose per un apprendente straniero c'è, senza dubbio, il sistema verbale dell'italiano che si caratterizza, tra l'altro, per una complicata morfologia flessiva, non solo per quanto riguarda il numero di modi e tempi verbali che lo studente deve imparare, ma anche per quanto concerne un abbondante numero di forme flesse irregolari di cui è ricco questo sistema. Con il presente articolo si cerca di esaminare come vengono presentate le irregolarità flessive del verbo nelle nuove grammatiche per stranieri (sono stati analizzati 18 volumi pubblicati a partire dal 2001 che si riferiscono al Quadro Comune Europeo di Riferimento). L'analisi si concentra sulla coniugazione dei tempi passati: imperfetto, passato prossimo (ci si concentrerà sulle forme del participio passato, per cui gli altri tempi passati composti rimangono fuori interesse) e passato remoto. Oltre a una dettagliata analisi comparativa dei volumi presi in esame, al termine di ogni paragrafo viene fatta una proposta didattica sulla presentazione di tali forme flesse.

Parole chiave: italiano L2/LS, insegnamento della grammatica, didattica della lingua italiana L2/LS, flessione verbale, verbi irregolari.

\section{INTRODUZIONE}

La morfologia flessiva della lingua italiana è particolarmente complessa: nonostante che la declinazione in italiano riguardi solo il numero e il genere (e il caso morfologico è oggi assente o - più precisamente - si manifesta in maniera molto limitata con i pronomi personali e i pronomi relativi), il verbo italiano, invece, si coniuga in sette modi, tra cui quattro sono finiti (indicativo, congiuntivo, condizionale e imperativo), quindi

\footnotetext{
*daniel.slapek@uwr.edu.pl
} 
richiedono diversi morfemi grammaticali a seconda della persona e del numero. In tal modo, un solo verbo può avere sei desinenze per un solo modo e tempo verbale (p. es. al presente indicativo, per i verbi della prima coniugazione: $-o,-i,-a$, -iamo, -ate, -ano), o addirittura nove desinenze, se si tratta del passato remoto dei verbi regolari uscenti in -ere (-ei/-etti, -esti, -é/-ette, -emmo, -este, -erono/-ettero). Ma il numero di forme flesse - sempre per un solo modo e tempo verbale - può essere anche più alto, fino ad arrivare a dieci (alcuni verbi hanno forme alternative quanto all'infisso -isc- del presente indicativo, come mentire: mento/mentisco, menti/mentisci ecc.; cfr. Sensini 2011: 303) o addirittura a dodici (ci sono verbi che hanno due forme alternative della radice, $\mathrm{p}$. es. sedere o udire al futuro indicativo: sederò/siederò, udirò/udrò ecc.; cfr. Stoppelli 2004: 80, 102).

Il sistema verbale italiano abbonda, inoltre, di forme flesse irregolari che - come se non bastasse - si alternano a volte con quelle regolari (p. es. i participi passati perso/perduto, visto/veduto ecc.). Una simile ricchezza morfologica può sicuramente scoraggiare uno studente straniero, perciò le dette irregolarità flessive gli andrebbero esposte in maniera possibilmente più ordinata, per non dire regolare. Basti pensare alle corrispondenze (quindi regolarità, per cui anche nel titolo leggiamo "(ir)regolarità flessive") che si riscontrano tra vari gruppi di verbi (p. es. alcuni participi passati hanno la stessa terminazione: dire $>$ detto; fare $>$ fatto; cuocere $>$ cotto) o tra varie categorie grammaticali (p. es. numerosi verbi che al participio passato finiscono in -sto al passato remoto terminano in -si: nascondere $>$ nascosto $>$ nascosi; proporre $>$ proposto $>$ proposi; rimanere $>$ rimasto $>$ rimasi). Purtroppo, come vedremo in seguito, gli autori delle grammatiche per stranieri sono poco disposti a ritrovare tali corrispondenze (e quindi a facilitare lo studio) e spesso si limitano e redigere una lista dei verbi irregolari relativa a un dato modo e tempo, rendendo in effetti lo studio della grammatica italiana più difficile.

Con il presente articolo si cerca di esaminare, per l'appunto, come le nuove grammatiche d'italiano $\mathrm{L}^{2} / \mathrm{LS}^{1}$ presentano le irregolarità flessive del verbo. Per motivi di spazio, mi limiterò ai tempi passati dell'indicativo: imperfetto (d'ora in poi indicato anche come IMP; a questo proposito esaminerò anche l'esposizione delle desinenze regolari che varia da titolo a titolo), passato remoto (PR) e passato prossimo (PPros) (chiaramente si tratta delle forme irregolari del participio passato (PPass); tuttavia la maggior

${ }^{1}$ In termini generali, si tratta di insegnamento dell'italiano come lingua non materna, quindi sia come lingua straniera, sia come lingua seconda, perciò i due termini sono in questa sede intercambiabili. Le grammatiche analizzate si usano, per l'appunto, nei due contesti didattici (per le definizioni di italiano lingua materna, seconda, straniera e etnica si veda p. es. Balboni 2014: 17-23). 
parte delle grammatiche per stranieri presenta tali forme nei capitoli dedicati al PPros, perciò ricorro a questa metonimia; rimangono fuori dal mio interesse $\mathrm{i}$ tempi passati composti in quanto formati da un verbo ausiliare seguito da un PPass $)^{2}$. Il testo si articola in paragrafi che corrispondono ai tre tempi passati di cui sopra. Nell'appendice vengono, inoltre, aggiunte le tabelle che confrontano il numero di tutti i verbi irregolari presentati nelle grammatiche esaminate.

Sono stati analizzati venti volumi pubblicati dalle case editrici più note nell'ambito dell'insegnamento dell'italiano come lingua non materna, di seguito elencati. I titoli sono esposti in ordine alfabetico a seconda delle sigle che, per comodità, verranno usate nel corso del testo; nelle parentesi quadre si riportano i livelli QCER cui è dedicato un dato titolo:

- CI: Comunicare in italiano [A1/C1] (Chiuchiù \& Chiuchiù 2015);

- GAt: Grammatica attiva [A1/B2+] (Landriani 2012);

- GB: Grammatica di base [A1/B2] (Esposito \& Errico 2007);

- GBI: Grammatica di base dell'italiano [A1-B1] (Petri et al. 2015);

- GdU: Grammatica d'uso della lingua italiana [A1/B2] (Celi \& La Cifra 2011);

- GeP: Grammatica e pratica [A2-B1] (Colombo 2006);

- GiC: Grammatica in contesto [A1/B1] (Gatti \& Peyronel 2006);

- G.it: Gramm.it [A1/C1] (Iacovoni et al. 2009);

- GL2: Grammatica dell'italiano L2 [A1-C2] (Duso 2019)

- GP: Grammatica pratica della lingua italiana [A1/C1] (Mezzadri 2016);

- GS1: Grammatica della lingua italiana Per Stranieri, vol. 1: di base [A1-A2] (Tartaglione \& Benincasa 2015a);

- GS2: Grammatica della lingua italiana Per Stranieri, vol. 2: intermedio-avanzato [B1-B2] (Tartaglione \& Benincasa 2015b);

- GT1: Una grammatica italiana per tutti 1, vol. 1: livello elementare [A1/A2] (Latino \& Muscolino 2014a);

- GT2: Una grammatica italiana per tutti 1 [B1/B2] (Latino \& Muscolino 2014b);

- IE: Italiano essenziale [A1-B2] (Mezzadri 2003);

- LSR: La lingua italiana e le sue regole [A1-B2] (Debetto 2016);

- NGP: Nuova grammatica pratica della lingua italiana [A1/B2] (Nocchi 2011);

${ }^{2}$ I due tempi perfetti vengono anche chiamati passato semplice e composto (cfr. p. es. Squartini 2010: 519, 524); eppure, tutte le grammatiche per stranieri qui riferite preferiscono la coppia terminologica passato prossimo e passato remoto. 
- UD1: L'utile e il dilettevole 1 [A1/B1] (Ercolino \& Pellegrino 2011);

- UD2: L'utile e il dilettevole 2 [B2/C2] (Ercolino e Pellegrino 2012);

- VG: Via della grammatica [A1/B2] (Ricci 2011).

Nel paragrafo relativo all'imperfetto indicativo e al passato prossimo non verranno presi in considerazione i volumi GS2, GT2 e UD2, dedicati alla fascia $\mathrm{B}$ del QCER (l'IMP e il PPros vengono introdotti ai livelli più bassi: A1/A2; cfr. Cesarini 2007: 139, 145); invece dal paragrafo relativo al passato remoto sono esclusi GS1, GT1 UD1 in quanto destinati ai livelli in cui questo tempo verbale non si insegna ancora (le forme regolari e irregolari del PR vengono solitamente introdotte a partire dal livello B2; ivi: 161) ${ }^{3}$.

\section{IMPERFETTO INDICATIVO}

L'imperfetto dell'indicativo è senz'altro il tempo verbale che si caratterizza per la minore irregolarità nella coniugazione, le cui desinenze coincidono in tutti e tre i paradigmi verbali. Nonostante una semplice, per così dire, morfologia flessiva, la presentazione delle sue forme verbali risulta particolarmente variegata nelle grammatiche per stranieri. In tal modo, nei volumi esaminati in questa sede troveremo, per esempio, le sole desinenze verbali, -avo, -avi, -ava ecc., senza che si trovino alla fine di un dato verbo (è la soluzione scelta in UD1, dove - in più - vengono presentati separatamente tutti i paradigmi flessivi, quindi il numero di desinenze è addirittura triplicato; ivi: 158) o incorporate in un'intera forma flessa. Nel secondo caso, 1) la desinenza grammaticale viene presentata per intero, insieme alla vocale tematica del verbo; si presentano, quindi, $i$ tre paradigmi flessivi in parallelo (o addirittura quattro: IE - come l'unico volume - presenta separatamente due tipi dei verbi uscenti in -ire, le cosiddette varianti $a$ e $b$ della $3^{a}$ coniugazione: "IIIa senti-vo, IIIb fin-ivo"; ivi: 74); tale scelta sembra sconsigliabile in quanto moltiplica il numero di desinenze che l'apprendente deve affrontare $;{ }^{4} 2$ ) la desinenza è priva della vocale tematica, per cui viene presentato un unico paradigma flessivo per tutti i verbi; p. es. CI (p. 113)

${ }^{3}$ Nell'analisi non mi riferirò ai due volumi progettati per i livelli più alti: Grammatica avanzata della lingua italiana (Nocchi \& Tartaglione 2006) e GrammaticAvanzata (Troncarelli \& La Grassa 2017) perché - anche se conformi al QCER - non presentano le forme verbali come tali.

${ }^{4}$ Inoltre, la stessa desinenza viene evidenziata in vari modi: a) con dei colori diversi (VG: 116), b) in grassetto (GB: 180; GBI: 151; GeP: 98; G.it: 149; GT1: 105), c) con un trattino: am-avo, ved-evo, apr-ivo (NGP: 114; similmente in GAt: 175; GL2: 136; GP: 68). 
in una sola tabella introduce sia verbi regolari che irregolari ${ }^{5}$ (troveremo una simile soluzione in GdU, dove dalla forma flessa viene ulteriormente separata la vocale tematica: $a m-a-v o$, vend-e-vo, sent-i-vo; ivi: 100); 3) la vocale tematica e la marca del passato $-v$ - vengono separate dal morfema indicante la persona e il numero: am-av-o, tem-ev-o, part-iv-o ecc.; è la soluzione proposta in LSR (p. 73) che, però, pare forse poco opportuna perché è una via di mezzo tra una morfologia semplificata (radice + desinenza, $\mathrm{p}$. es. am-are) e una vera e propria struttura morfologica dell'IMP: radice del verbo + vocale tematica $(-a-,-e-,-i-)+$ marca temporale/modale $(-v-)+$ desinenza personale (cfr. Palermo 2015: 159).

Tra i verbi adoperati per esemplificare la coniugazione regolare troviamo soprattutto verbi che sono regolari anche al presente indicativo, tra gli altri: $i$ ) per la $1^{\text {a }}$ coniugazione: parlare (il più frequente), amare, cantare; ii) per la $2^{\text {a }}$ coniugazione: vivere, vedere, prendere; iii) per la $3^{\text {a }}$ coniugazione: aprire, sentire, dormire ecc. È interessante servirsi a questo punto delle forme solitamente irregolari negli altri tempi o modi verbali (specie al presente indicativo, che è già noto agli apprendenti). Tale soluzione è stata proposta solo in GAt (p. 175), che adopera andare per il primo gruppo di verbi, nonché in GS1 (p. 92), che presenta avere per il secondo gruppo (ciò permette di evitare di costruire una tabella a parte con i soliti due verbi irregolari avere ed essere, com'è invece stato fatto in GP: 68; G.it: 149; GeP: 98). Similmente, si rivela una soluzione brillante servirsi - per esemplificare il terzo paradigma flessivo - dei verbi che al presente indicativo richiedono l'infisso -isc- (p. es. capire in VG: 16; GB: 180; preferire in GAt: 175 ; in tal modo si scioglie subito il dubbio su un'eventuale differenza nella coniugazione di questi verbi).

Alcuni volumi, accanto agli esempi di coniugazione, aggiungono diverse note relative alla formazione dell'IMP. Queste possono essere $i$ ) piuttosto semplici, p. es. "Alla prima parte del verbo, che non cambia, aggiungiamo le varie desinenze" (VG: 116; una simile nota pare ridondante se è posta accanto alla tabella in cui, con un diverso colore, vengono evidenziate tutte le desinenze) o $i i$ ) più precise, p. es. "Le desinenze -vo, -vi, -va, -vamo,-vate, -vano valgono per tutti i gruppi di verbi. Cambia la vocale che le precede [...]" (GeP: 98; similmente in GB: 180; i due testi risultano incoerenti, perché negli esempi che proongono sono evidenziati in grassetto le stesse desinenze insieme alla vocale tematica, quindi -avo, $-e v o,-i v o)^{6}$, accompagnate anche

${ }^{5}$ In questo caso le irregolarità riguardano, chiaramente, soltanto le radici del verbo.

${ }^{6}$ Un simile commento troviamo anche in GdU (p. 100) dove, tuttavia, viene curiosamente suggerito di dovere "aggiungere" la vocale tematica alla radice verbale: "L'imperfetto si forma aggiungendo al tema del verbo, prima delle desinenze, che sono uguali per le tre coniugazioni, la vocale tematica $[\ldots]$ ".. 
dalle informazioni sulla morfologia del verbo, p. es. "[l'IMP] è formato da: radice dell'infinito $+-a v$ - (per la $1^{\text {a }}$ coniugazione) [ecc.] + desinenze" (LSR: 73 ; da ciò si evince che i morfemi grammaticali sono rispettivamente $-o,-i$, -a,-amo, -ate, -ano, per cui sarebbe forse lecito precisare che tali desinenze coincidono, nella maggior parte, con quelle del presente indicativo dei verbi uscenti in -are). In uno dei volumi analizzati troveremo, inoltre, una nota di natura mnemotecnica: "Per aiutare a memorizzare la forma dell'imperfetto, e distinguerlo dagli altri e modi verbali, può essere utile notare la presenza caratteristica nella coniugazione della lettera $v$ " (GT1: 107; in questo caso gli autori cercano di evitare una terminologia tecnica che abbiamo visto negli esempi precedenti).

Sono pochi i verbi irregolari all'IMP: essere, bere, dire, fare, porre, trarre e i loro composti, nonché i composti di -durre. I primi quattro vengono effettivamente presentati nella maggior parte delle grammatiche, il meno frequente è invece trarre, esposto solo in sei volumi (si veda la tabella 4 dell'appendice; incuriosisce il caso di LSR con un solo verbo irregolare essere) ${ }^{7}$. Alcuni autori aggiungono anche diversi commenti sulla provenienza latina delle irregolarità tematiche, p. es. 1) "[ci sono] verbi che formano l'imperfetto sull'infinito latino: i più importanti sonofacevo (da facere), dicevo (da dicere), bevevo (da bibere)" (GL2: 137); 2) "[...] bere, dire, fare, e i verbi in-arre,-orre, -urre formano l'imperfetto sulla base del loro infinito latino: bevere, dicere, facere, estra(h) ere, proponere, traducere ecc." (GB: 180); $3)$ " $i$ verbi in -durre, composti di un verbo *durre che però in italiano non esiste, derivato dal verbo latino ducere" (GS2: 88). Non mancano, inoltre, indicazioni poco precise, come p. es. "I verbi che finiscono in -urre [...] aggiungono la - $c$ - prima della vocale tematica" (GdU: 102; gli apprendenti che non hanno avuto una solida preparazione linguistica potrebbero avere difficoltà a ricostruire tali forme flesse), o addirittura commenti infondati: "[...] qualche irregolarità importante si trova solo nella coniugazione del verbo essere e dei verbi dire, bere, fare" (GS1: 92; come abbiamo detto, tra gli irregolari si annoverano anche altri verbi).

Riassumendo quanto finora detto, credo che - per scopi didattici - sia opportuno $i$ ) limitare la desinenza dell'IMP alla parte priva della vocale tematica (in tal modo verrà palesemente indicato un solo paradigma flessivo per tutti i verbi), preferibilmente tramite verbi irregolari al presente indi-

${ }^{7}$ S'incontrano inoltre diversi prefissati di porre, trarre e -durre (cfr. tabella 4). La scelta potrebbe essere condizionata dalla frequenza d'uso di un dato verbo; a questo proposito si vedano i dati ricavati dal Corpus e Lessico di Frequenza dell'Italiano Scritto (cfr. Bertinetto et al 2005) nel cui lemmario proporre ha il rango d'uso 384 (è il più frequente tra i prefissati di porre) seguito da porre (rango d'uso 617) e opporre (2387); similmente, trarre (1475) precede estrarre (2826); invece tradurre (2276) segue produrre (583). 
cativo, p. es. andare per la $1^{\text {a }}$ coniugazione, avere per la $2^{\text {a }}$ coniugazione e un verbo uscente in -isc-al presente per il $3^{\circ}$ gruppo di verbi (in tal modo si eviteranno tutti gli eventuali dubbi che possono suscitare simili verbi); ii) indicare expressis verbis tutti i verbi che subiscono l'alternanza tematica, con eventuali commenti sulla loro etimologia latina e sulle loro forme composte; iii) per i verbi irregolari porre, trarre e -durre, servirsi dei loro prefissati più frequenti (cfr. tabella 1; per motivi di stampa sono scritti in grassetto frammenti che sarebbero preferibilmente evidenziati con un colore diverso da quello adoperato nel corpo del testo).

Tabella 1. Forme verbali dell 'imperfetto indicativo: una proposta di presentazione

\begin{tabular}{cc|cc|cc}
\hline \multicolumn{2}{c|}{ Verbi regolari } & \multicolumn{2}{c|}{6 verbi che cambiano il tema } & 1 verbo irregolare \\
\hline -are $(1)$ & anda-vo & bere & beve-vo & essere & ero \\
andare & anda-vi & dire & dice-vi & eri \\
-ere $(2)$ & ave-va & fare & face-va & era \\
avere & ave-vamo & proporre & propone-vamo & eravamo \\
-ire (3) & fini-vate & produrre & produce-vate & & eravate \\
finire & fini-vano & trarre & trae-vano & & erano \\
\hline
\end{tabular}

\section{PASSATO PROSSIMO (PARTICIPIO PASSATO)}

Come si è già detto, le forme del participio passato (regolari e irregolari) vengono solitamente introdotte nei capitoli dedicati al passato prossimo. Due volumi hanno dedicato al participio un capitolo a parte (GeP: 192-194, dove si parla del participio sia passato che presente; GB: 159, dove il capitolo sul participio passato precede immediatamente quello sul passato prossimo). Inoltre, GdU (pp. 92-93) prevede un apposito capitolo dedicato alle irregolarità del PPass ("Il passato prossimo indicativo irregolare", dove però si limita al solo elenco dei verbi irregolari), invece in GBI non si presentano affatto tali irregolarità .

Quanto ai participi irregolari, la soluzione più frequente è - purtroppo - quella di presentare una singola lista, disposta solitamente in ordine alfa-

${ }^{8}$ Alla fine del volume troviamo le tavole di coniugazione con le forme flesse di tutti i modi e tempi verbali di alcuni verbi irregolari più frequenti: essere, avere, stare, dare, fare, dire, sapere, potere, volere, andare, uscire, bere, rimanere, venire (GBI: 272-280; tra i verbi irregolari vengono annoverati anche: cercare, pagare, cominciare, mangiare). 
betico ${ }^{9}$ senza indicarne alcune regolarità, per così dire, interne (si vedano GAt: 131-132; GdU: 92; GL2: 129-130; GP: 64; GS1: 64; IE: 96; LRS: 98; NGP: 80; UD1: 90-91; VG: 102-103 ${ }^{10}$; la più numerosa risulta la lista di 72 verbi presentata in UD1 ${ }^{11}$ ). Tra i verbi irregolari esposti nelle grammatiche troviamo inaspettatamente 1) alcuni verbi regolari al PPass: ottenere (GeP: 193), stare (NGP: 80; VG: 102), conoscere (GdU: 92; GeP: 193) e piacere (IE: 96; GeP: 193; GL2: 129; GP: 64; VG: 102); gli ultimi due andrebbero classificati come particolarità della coniugazione regolare in quanto i verbi in -cere e -scere "aggiungono una $i$ prima della terminazione" (GiC: 94; $\mathrm{GiC}$ è l'unico volume che tiene in considerazione questo gruppo di verbi); 2) diverse forme prefissate che si coniugano al PPass come il verbo base (la più ricca da questo punto di vista è $\mathrm{GeP}(\mathrm{p}$. 193) il cui elenco comprende $\mathrm{i}$ seguenti prefissati: (ri-)chiedere, (per-)correre, (con-)dividere, (pro-)muovere, (rim-)piangere, (com-, dis-, es-, im-)porre, (com-)prendere, (at-, pre-) tendere, (pre-)vedere; invece di allargare un lungo elenco di irregolarità, basterebbe una semplice nota relativa ai verbi composti). Per una panoramica dei verbi irregolari che troviamo esposti nelle grammatiche analizzate si veda la tabella 5 dell'appendice ${ }^{12}$.

L'unica sottoclasse di verbi di cui si tiene conto (ma solo in tre volumi) sono i verbi che terminano in -arre, -orre, -urre e che "hanno i participi rispettivamente in -atto, -osto, e -otto" (GeP: 193). Tuttavia, GB ne indica solo due gruppi: -arre e -urre (cui accosta inaspettatamente i verbi in -cere >-ciuto; ivi: 159), invece GiC si limita ai soli verbi in -urre (p. 94;

${ }^{9}$ Negli elenchi alfabetici è facile trovare alcune sviste nell'ordine, p. es. scrivere, scoprire (GS1: 64); essere, esprimere (Gat: 131); spingere, soffrire, sorprendere (UD: 91). Inoltre, alcuni titoli indicano forme flesse alternative: perdere: perso o perduto (GB: 159; IE: 96); vedere: visto o veduto (ibidem), tra cui solo una grammatica aggiunge un apposito commento: "perdere e vedere hanno anche una forma regolare (perduto, veduto) che, però, si usa raramente" (GiC: 94).

${ }^{10}$ G.it (p. 108) divide la sua lista in due parti: verbi che si coniugano con l'ausiliare essere e avere; GeP (p. 193) divide i verbi in quelli adatti al livello A2 (es. bere, dire, vivere) e B1 (concedere, muovere, tendere ecc.); non troviamo invece alcuna indicazione sulle corrispondenze tra le desinenze dell'infinito e del PPass. CI risulta a questo proposito piuttosto disordinata: nonostante ci sia una tabella con i participi irregolari (ivi: 53), essa non include numerosi verbi che invece sono stati esposti in altre tabelle, p. es. venire si trova nella parte dedicata alla coniugazione con l'ausiliare essere (ivi: 54), vivere si trova nella tabella con i verbi che "hanno indifferentemente essere o avere: vivere, campare, durare" (ivi: 57) ecc.

${ }^{11}$ Seguono le liste di 50 verbi (GAt: 131-132), di 40 verbi (IE: 96; GP: 64; VG: 102-103) o di 35 verbi irregolari (GL2: 129-130).

${ }^{12}$ Come vedremo, tra i 114 verbi in totale, 49 verbi sono stati presentati da un solo volume. 
tale scelta è del tutto comprensibile perché i participi dei verbi trarre e porre sono indicati come irregolari, per cui tutti i loro composti avranno la stessa terminazione). Invece l'unico titolo che ha diviso in gruppi la lista dei participi irregolari è GT1, dove troviamo le seguenti terminazioni: 1) -tto (correggere, dire, fare e altri); 2) -so (accendere, chiudere, decidere ecc.); 3) -sto (chiedere, proporre, rimanere ecc.); 4) -rto (aprire, morire, offrire ecc.); 5) -sso (discutere, mettere, permettere ecc); 6) -nto (dipingere, piangere, vincere ecc.); 7) -uto (bere, venire, vivere ecc.), più un gruppo eterogeneo con i seguenti verbi: ridurre, tradurre, perdere, scegliere. Una simile soluzione sembra interessante, ma potrebbe essere migliorata-come credo - se venissero indicate anche altre corrispondenze strutturali.

Proporrei pertanto di evidenziare le seguenti classi di verbi che solitamente hanno una terminazione dell'infinito e del PPass in comune (cfr. tabella 2, dove vengono indicate anche altre irregolarità): $i$ ) l'infinto uscente in -dere $\mathrm{e}$ -ndere cambia al PPass in -so (es. chiudere > chiuso; accendere > acceso ecc.); ii) -ngere cambia in -nto (es. dipingere $>$ dipinto); iii) -gliere cambia in -lto (es. cogliere $>$ colto); $i v)$-rgere cambia in -rso (es. emergere $>$ emerso) oppure in -rto (accorgere $>$ accorto); v) -ggere cambia in -tto (es. correggere $>$ corretto ${ }^{13}$; vi) -durre cambia in -dotto (es. produrre $>$ prodotto). Si potrebbero, inoltre, indicare alcuni gruppi di verbi meno numerosi (nella parte $c$ della tabella 2 tali verbi sono indicati come particolari; ciò per non aumentare il numero di desinenze dell'infinito di cui l'apprendente dovrebbe tenere conto), dove vii) -stere cambia in -stito (es. assistere > assistito); viii) -volvere cambia in -voluto (evolvere $>$ evoluto); $i x)$-solvere cambia -solto (risolvere $>$ risolto $) ; x$ )-primere cambia in -presso (esprimere > espresso); xi) -mere cambia in -nto (assumere $>$ assunto); xii) -parire cambia in -parso (apparire > apparso).

A tali classi di verbi andrebbero aggiunti i participi passati irregolari, che non si possono dedurre dalla forma dell'infinito (nella parte $b$ della tabella 2 ci sono 32 verbi, raggruppati in più a seconda della terminazione, tra cui i verbi non presentati da nessuna delle grammatiche esaminate: fondere $>$ fuso, prediligere $>$ prediletto, scuotere $>$ scosso, valere $>$ valso). Inoltre, andrebbe detto in un'apposita annotazione che i verbi composti si coniugano di solito come il verbo base (correre $>$ corso, intercorrere $>$ intercorso; percorrere $>$ percorso ecc.), anche se ci sono diverse eccezioni a questa regola ( p. es. cedere $>$ ceduto, procedere $>$ proceduto, ma concedere $>$ concesso ecc.).

${ }^{13}$ Non c'è bisogno di indicare altri due gruppi di verbi uscenti in -lgere o -ncere perché, come risulta dal lemmario di CoLFIS, tutti i verbi uscenti in -lgere sono prefissati di volgere (p. es. avvolgere, coinvolgere, sconvolgere, travolgere ecc.), invece tutti i verbi in -ncere sono prefissati di vincere (p. es. avvincere, convincere, evincere, stravincere ecc.). Pertanto, basta indicare il verbo base tra i participi passati irregolari. 
Tabella 2. Forme irregolari del participio passato: una proposta di presentazione

a) Corrispondenze infinito - participio passato

\begin{tabular}{|c|c|}
\hline \multirow{3}{*}{$\begin{aligned} \text {-DERE } & \rightarrow \text {-SO } \\
\text { chiudere } & \rightarrow \text { chiuso }\end{aligned}$} & $\begin{array}{l}\text { Esempi: } \\
\text { concludere: concluso } \\
\text { decidere: deciso }\end{array}$ \\
\hline & $\begin{array}{l}\text { a) alcuni verbi in -dere sono regolari (-duto): } \\
\text { p. es.: cadere, cedere, credere, presiedere }\end{array}$ \\
\hline & $\begin{array}{l}\text { b) alcuni participi dei verbi in -dere non si possono dedurre } \\
\text { dalla forma dell'infinito } \\
\text { p. es.: chiedere, succedere, vedere }\end{array}$ \\
\hline \multirow{3}{*}{$\begin{aligned} \text {-NDERE } & \rightarrow-\text { SO } \\
\text { prendere } & \rightarrow \text { preso }\end{aligned}$} & $\begin{array}{l}\text { Esempi: } \\
\text { accendere: acceso } \\
\text { difendere: difeso }\end{array}$ \\
\hline & $\begin{array}{l}\text { a) alcuni verbi in -ndere sono regolari (-nduto) } \\
\text { p. es.: vendere }\end{array}$ \\
\hline & $\begin{array}{l}\text { b) alcuni verbi in -ndere sono irragolari } \\
\text { p. es.: fondere, nascondere, rispondere }\end{array}$ \\
\hline \multirow[t]{2}{*}{$\begin{aligned}-\mathrm{NGERE} & \rightarrow-\mathrm{NTO} \\
\text { piangere } & \rightarrow \text { pianto }\end{aligned}$} & $\begin{array}{l}\text { Esempi: } \\
\text { aggiungere: aggiunto } \\
\begin{array}{ll}\text { dipingere: dipinto } & \text { pungere: giunto }\end{array}\end{array}$ \\
\hline & Eccetto: stringere: stretto \\
\hline $\begin{aligned}- \text { gliere } & \rightarrow \text {-lto } \\
\text { scegliere } & \rightarrow \text { scelto }\end{aligned}$ & Esempi: cogliere, raccogliere, sciogliere, togliere \\
\hline $\begin{aligned}- \text { RGERE } & \rightarrow-\text { RSO } \\
\text { spargere } & \rightarrow \text { sparso }\end{aligned}$ & Esempi: convergere, emergere, immergere, sommergere, tergere \\
\hline $\begin{array}{l}\text {-RGERE } \rightarrow-\text { RTO } \\
\text { sorgere } \rightarrow \text { sorto }\end{array}$ & Esempi: accorgere, porgere, sorgere, sporgere, risorgere, scorgere \\
\hline $\begin{aligned} \text {-GGERE } & \rightarrow \text {-TTO } \\
\text { leggere } & \rightarrow \text { letto }\end{aligned}$ & Esempi: correggere, distruggere, friggere, proteggere \\
\hline $\begin{aligned} \text {-DURRE } & \rightarrow \text {-DOTTO } \\
\text { produrre } & \rightarrow \text { prodotto }\end{aligned}$ & Esempi: condurre, ridurre, tradurre, dedurre \\
\hline
\end{tabular}


b) Participi passati che non si possono dedurre dalla forma dell'infinito

\begin{tabular}{|c|c|c|}
\hline \multirow{5}{*}{$\begin{array}{l}\text { chiedere: chiesto } \\
\text { nascondere: nascosto } \\
\text { porre: posto } \\
\text { rispondere: risposto } \\
\text { vedere: visto }\end{array}$} & fare: fatto & aprire: aperto \\
\hline & trarre: tratto & coprire: coperto \\
\hline & dire: detto & morire: morto \\
\hline & prediligere: prediletto & offrire: offerto \\
\hline & stringere: stretto & \\
\hline & cuocere: cotto & correre: corso \\
\hline discutere: discusso & rompere: rotto & fondere: fuso \\
\hline mettere: messo & scrivere: scritto & valere: valso \\
\hline muovere: mosso & & vivere: vissuto \\
\hline scuotere: scosso & essere: stato & volgere: volto \\
\hline & nascere: nato & \\
\hline distinguere: distinto & bere: bevuto & \\
\hline vincere: vinto & venire: venuto & \\
\hline
\end{tabular}

c) Altri verbi particolari

\begin{tabular}{cl}
\hline assistere $\rightarrow$ assistito & Esempi: esistere, insistere, persistere \\
\hline esprimere $\rightarrow$ espresso & Esempi: deprimere, imprimere \\
\hline assumere $\rightarrow$ assunto & Esempi: desumere \\
\hline risolvere $\rightarrow$ risolto & Esempi: assolvere, dissolvere \\
\hline \multirow{2}{*}{ evolvere $\rightarrow$ evoluto } & Esempi: devolvere, involvere \\
& Eccetto: volvere (volto) \\
\hline \multirow{2}{*}{ apparire $\rightarrow$ apparso } & Esempi: apparire, comparire, scomparire, trasparire \\
& Eccetto: sparire (sparito) \\
\hline
\end{tabular}

\section{PASSATO REMOTO}

Il passato remoto "costituisce uno dei tempi più complessi del paradigma verbale" (D’Achille 2003: 121). La sua complessità si manifesta i) con una particolare alternanza d'uso con il PPros (dove entrano in gioco sia fattori puramente linguistici ${ }^{14}$ sia fattori sociolinguistici ${ }^{15}$ e geografici ${ }^{16}$ ),

${ }^{14}$ Come l'effettiva lontananza temporale o il legame dell'avvenimento al presente ( $\mathrm{p}$. es. Berretta 1993: 210); inoltre, il PR "è contraddistinto dai seguenti tratti: a) conclusione del processo anteriormente al momento dell'enunciazione; b) deitticità della designazione temporale; c) specificità del riferimento temporale, che non tollera interpretazioni eventuali, abituali, e simili" (Bertinetto 2001: 99).

${ }^{15}$ Tra i quali Squartini (2015: 49) indica il grado di formalità del contesto comunicativo, la distinzione scritto e parlato, il tipo di testo, nonché il grado della sua elaborazione letteraria.

${ }^{16}$ Nella varietà settentrionale parlata (o nello scritto informale) il PR è in disuso (cfr. Berretta 1993: 210); sulla distribuzione dei diatopica due tempi passati si veda p. es. Bertinetto e Squartini (1996). 
ii) con una ricca morfologia flessiva che - dal punto di vista sincronico - presenta due principali problemi: a) il paradigma arizotonico simmetrico in -ei/-etti (il PR è l'unico tempo verbale che ammette due serie di desinenze regolari) ${ }^{17}, \mathrm{~b}$ ) le alternanze tematiche dei tipi rizotonici a cui viene dedicato questo paragrafo (le forme flesse arizotoniche/rizotoniche sono dette anche deboli/forti o, nelle grammatiche normative e - soprattutto - quelle didattiche per stranieri, regolari/irregolari; cfr. Schwarze 2009: 98-99; Tekavčić 1980: 298) ${ }^{18}$.

Sorprende quanto risultino disordinate le grammatiche d'italiano L2/ LS nel presentare la coniugazione delle forme rizotoniche. In generale, gli autori esplicano che l'irregolarità riguarda solo le forme della $1^{\mathrm{a}}$ e della $3^{\mathrm{a}}$ persona singolare, nonché della $3^{\text {a }}$ persona plurale (p. es. GS2: 162, cfr. NGP: 225, VG: 222) ${ }^{19}$, con le rispettive desinenze $-i$, -e, -ero (fanno eccezione $\mathrm{i}$ verbi essere, dare, stare), quindi "per coniugare i verbi irregolari [in realtà] è necessario ricordare la forma della prima persona" (GS2: 162), tuttavia sono pochi i volumi che cercano di esporre l'allomorfia tematica del PR in maniera sistematica.

Alcuni autori provano a raggruppare i verbi, soprattutto in base all'infinito, p. es. 1) GB (p. 174) ne trova due classi : a) "i verbi nascere, tacere e piacere formano il passato prossimo seguendo lo stesso schema" e b) "alcuni verbi [...] si rifanno al loro infinito latino" (gli esempi che dà sono: bevere, dicere, facere, opponere, traducere, producere, conducere, estra(h)ere; GB è l'unico titolo che tiene conto di questo gruppo di verbi);2) $\mathrm{GeP}$ (p. 130) mette in evidenza i verbi in -arre, orre, -urre (particolarmente insidiosi perché hanno sia le forme irregolari trassi, trasse, trassero ecc. sia

${ }^{17}$ In realtà, oggi prevalgono le desinenze lunghe di tipo -etti e la presunta intercambiabilità delle due serie dei morfemi - suggerita dalle grammatiche - è solo teorica (per uno studio sulla falsa alternanza tra le forme verbali uscenti in -ei, -é,-erono ed -etti,-ette, -ettero si veda Słapek 2020).

${ }^{18}$ Capita - purtroppo - che negli elenchi dei verbi irregolari ci siano anche le forme deboli, p. es. dovei/dovetti (in GS2: 163).

${ }^{19}$ Eventualmente, in un'altra prospettiva: "la $2^{\mathrm{a}}$ persona singolare, la $1^{\mathrm{a}} \mathrm{e}$ la $2^{\mathrm{a}}$ persona plurale sono quasi sempre regolari" (GB: 174) oppure "il passato remoto irregolare si forma aggiungendo le desinenze e due temi: vid-i, ved-esti, vid-e, ved-emmo, ved-este, vid-ero" (GdU: 124). Ciò a volte viene detto in maniera poco precisa, p. es. "spesso sono irregolari la $1^{\text {a }}$ e la $3^{\text {a }}$ persona singolare e la $3^{\text {a }}$ persona plurale" (GeP: 129; il corsivo è mio); in altri titoli si parla a questo proposito di "moltissimi verbi" (GT2: 70), de "la maggioranza dei verbi" (GBI: 144) o della "maggior parte dei casi" (IE: 78, GP: 93); invece, se a chiare lettere vengono esposti i tre verbi del tutto irregolari (come sopra), tale regola vale per tutte le altre forme flesse. Non mancano inoltre avvertimenti a dir poco imprecisi a questo riguardo: "essere e avere hanno una coniugazione propria" (LSR: 97; in questo caso avere non è un verbo del tutto irregolare). 
quelle latine: traesti, traemmo, traeste; a questo gruppo andrebbero quindi aggiunti i verbi bere, fare, dire, il che non è stato fatto; cfr. GB al primo punto); 3) UD2 (p. 2) pone l'attenzione su quattro desinenze dell'infinito: "Generalmente, i verbi che terminano in -dere, -cere, -gere e -gliere alla prima persona singolare e alla terza persona singolare e plurale prendono la s"; 4) GL2 (p. 153) aggiunge ancora tre desinenze: -ggere, -ndere, -gnere; inoltre, come suggerisce l'autrice, "ci sono verbi che raddoppiano la consonante finale della radice" (ivi: 154; ma è piuttosto un'osservazione e non una regola grammaticale vera e propria).

Inspiegabilmente, gli autori delle grammatiche per stranieri non parlano delle corrispondenze tra le forme del PR e quelle del PPass. Soltanto GiC divide i verbi in sei gruppi a seconda delle loro irregolarità, ma le regole che forma paiono insufficienti (tra parentesi alcuni miei commenti) ${ }^{20}$ : i verbi che 1) trasformano -so del PP in -si (preso $>$ presi); 2) trasformano la $t$ del PP in $s$ (dipinto $>$ dipinsi; nella proposta che segue se ne distingueranno due sottogruppi -nto e -lto); 3) trasformano il nesso consonantico st del PP in $s$ (chiesto $>$ chiesi); 4) trasformano il nesso $t t$ in $s s$ (letto $>$ lessi; a questo punto andrebbe detto che i verbi come produrre, con il PR produssi e non *prodossi, sono irregolari); 5) raddoppiano la consonante che precede la terminazione del PP (bevuto $>$ bevvi; tale regola è poco utile perché non indica quali verbi richiedono o meno il raddoppiamento consonantico); 6) nascere e piacere caratterizzati dalle consonanti $c q$ (a cui andrebbero aggiunti anche tacere e nuocere); e altri, irregolari (conobbi, ruppi ecc.; ivi: 292-296).

Sarebbe opportuno - a mio avviso - indicare in maniera precisa quali sono le dipendenze strutturali tra i due tempi passati. In tal modo avremo: 1) PP uscente in -so cambia al PR in -si (es. acceso $>$ accesi, corso $>$ corsi ecc.; fa eccezione solo parere: parso ma parvi);2) PP uscente in -nto cambia al PR in -nsi (es. finto $>$ finsi, pianto $>$ piansi); 3) PP uscente in -lto cambia al PR in -lsi (es. colto $>$ colsi, tolto $>$ tolsi) ${ }^{21}$; 4) PP uscente in -sto cambia al PR in -si (es. chiesto $>$ chiesi, nascosto $>$ nascosi; fa eccezione soltanto vedere: visto ma vidi), 5) PP uscente in -tto cambia al PR in -ssi (es. letto $>$ lessi, scritto > scrissi; fanno eccezione rompere: rotto ma ruppi, stringere: stretto ma strinsi, nonché i verbi uscenti in -durre, come produrre: prodotto ma produssi), 6) PP uscente in -sso cambia al PR in -ssi (es. mosso >

${ }^{20}$ Per essere precisi, anche GB accenna alla corrispondenza tra le forme del PPass e $\mathrm{PR}$, ma ne parla in maniera del tutto limitata: "molti verbi in -dere e tutti quelli in -ndere hanno forme simili a quelle del participio passato" (ivi: 173).

${ }^{21}$ I gruppi -lto, -nto (al PR -lsi, -nsi) sono trattati separatamente (diversamente da com'è proposto in $\mathrm{CiC}$ ) perché i verbi uscenti in -sto hanno al PR la desinenza -si o non

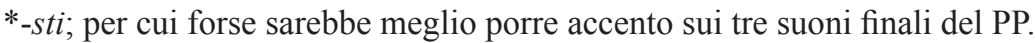


mossi; scosso > scossi; fa eccezione soltanto mettere: messo ma misi); 7) i verbi giacere, nascere, nuocere, piacere, tacere seguono lo stesso schema (nacqui, nascesti, nacque ecc.). Resta il gruppo dei verbi irregolari, ma ora ben preciso (17 verbi): bevvi ${ }^{22}$, caddi, conobbi, dissi, ebbi, feci, misi, parve, piovve, produssi (e altri composti con-durre), ruppi, seppi, strinsi, tenni, vidi, vissi, volli, e i loro derivati.

La stragrande maggioranza di testi propone una lista ordinata o meno (di solito in ordine alfabetico, cfr. p. es. GP: 93) di verbi appartenenti a tutti i paradigmi flessivi di cui sopra, aumentando in effetti il numero di forme irregolari che gli apprendenti devono imparare a memoria. Vince, per così dire, la tabella esposta in UD2 (pp. 1-2) dove si presentano addirittura 46 verbi (tra i quali mancano comunque le seguenti forme: caddi, misi, piovve, strinsi e volli, quindi un terzo dei verbi effettivamente irregolari $)^{23}$. La tabella 6 confronta il numero di verbi irregolari al PR esposti nelle grammatiche analizzate $^{24}$.

Riassumendo: per scopi didattici, la presentazione delle forme irregolari del PR andrebbe preferibilmente articolata come segue (cfr. tabella 3): i) i tre verbi irregolari, essere, dare ${ }^{25}$ e stare; ii) la coniugazione dei verbi irregolari alla $1^{\text {a }}$ e $3^{\text {a }}$ persona singolare e alla $3^{\text {a }}$ persona plurale; iii) le forme irregolari che non si deducono dalle forme del PPass (nella parte $b / 2$ della tabella, i verbi bere, dire, fare, -durre sono posti nella parte destra perché si trovano esplicati anche nella parte $e$ ); $i v$ ) le corrispondenze tra le forme del PPass e del PR; v) i verbi giacere, nascere, nuocere, piacere, tacere che seguono lo stesso schema, vi) i verbi le cui forme della $2^{\text {a }}$ persona singolare e della $1^{\text {a }}$ e $2^{\text {a }}$ persona plurale corrispondono a quelle latine.

${ }^{22}$ Due volumi presentano forme alternative del PR di bere: bevvi e bevetti (GBI: 144; GP: 93). Oggi, la seconda forma flessa è assente nei corpora dell'italiano scritto (cfr. Słapek 2016: 92).

${ }^{23}$ Seguono: BG (p. 175) con 38 verbi in una sola lista (mancano, tuttavia, stringere, piovere), CI (pp. 163-164) con 37 verbi (mancano parere, piovere, stringere), NGP (p. 226) con 33 verbi (mancano piovere, stringere, vivere, volere) e GAt (pp. 196-197) con 29 verbi (mancano piovere, stringere, nonché i verbi uscenti in -durre).

${ }^{24}$ I 22 verbi della tabella 6 sono esposti in un solo volume; i verbi più frequenti sono: essere, avere, venire (17 volumi), bere, dare, fare, sapere (16 volumi), nonché chiedere e vedere (15 volumi).

${ }^{25}$ La maggior parte delle grammatiche presenta due forme di dare: diedi e detti (sei volumi si limitano alla sola forma diedi; si veda tabella 6), anche se "la più comune e diffusa è la prima, che continua l'origine latino. Detti ha preso piede nel corso del Quattrocento [...]" (Della Valle \& Patota 2015: 145; uno studio dedicato alle forme diedi e detti è presentato in Patota 1987), come del resto altre forme arcaiche di cui è ricco il PR (p. es. aprilaperse, concedette/concesse, offri/offerse, perdette/perse ecc.; a questo proposito in Słapek 2016). 
Tabella 3. Irregolarità del passato remoto: una proposta

di presentazione

a) Verbi irregolari al passato remoto

\begin{tabular}{c|c|c}
\hline ESSERE & DARE & STARE \\
fui & diedi & stetti \\
fosti & desti & stesti \\
fui & diede & stemmo \\
fummo & demmo & steste \\
foste & deste & stettero \\
furono & diedero \\
\hline
\end{tabular}

b) Passato remoto irregolare (io, lui/lei, loro)

\begin{tabular}{c|ll|cc|ccc}
\hline \multirow{2}{*}{ 1) formazione } & \multicolumn{5}{|c}{ 2) verbi irregolari (desinenze: $-i,-e,-e r o$ ) } \\
\hline AVERE & CADERE & caddi & STRINGERE & strinsi & BERE & bevvi \\
ebbi & CONOSCERE & conobbi & TENERE & tenni & DIRE & dissi \\
avesti & METTERE & misi & VEDERE & vidi & FARE & feci \\
ebbe & PARERE & parve & VIVERE & vissi & PRODURRE & produssi \\
avemmo & PIOVERE & piovve & VOLERE & volli & & \\
aveste & ROMPERE & ruppi & & & & & \\
ebbero & SAPERE & seppi & & & & & \\
\hline
\end{tabular}

c) Corrispondenze tra le forme del Participio Passato e le forme del Passato Remoto

\begin{tabular}{c|c|c|c|c|l}
\hline PP & PR & Esempio: & PP & PR & Altri esempi: \\
\hline -so & - si & DIVIDERE & diviso & divisi & $\begin{array}{l}\text { accesi (ACCENDERE), chiusi (CHIUDERE), } \\
\text { offesi (OFFENDERE) }\end{array}$ \\
\hline -sto & - -si & CHIEDERE & chiesto & chiesi & $\begin{array}{l}\text { nascosi (NASCONDERE), proposi (PROPORRE), } \\
\text { rimasi (RIMANERE) }\end{array}$ \\
\hline -nto & $-n s i$ & VINCERE & vinto & vinsi & $\begin{array}{l}\text { dipinsi (DIPINGERE), piansi (PIANGERE), } \\
\text { spensi (SPEGNERE) }\end{array}$ \\
\hline- lto & -1 si & SCEGLIERE & scelto & scelsi & colsi (COGLIERE), tolsi (TOGLIERE) \\
\hline- tto & - ssi & LEGGERE & letto & lessi & diressi (DIRIGERE), scrissi (SCRIVERE), trassi (TRARRE) \\
\hline -sso & - ssi & MUOVERE & mosso & mossi & $\begin{array}{l}\text { discussi (DISCUTERE), rimOSSi (RIMUOVERE) } \\
\text { scossi (SCUOTERE) }\end{array}$ \\
\hline
\end{tabular}


d) Verbi con il nesso consonantico - $c q$ -

\begin{tabular}{c|c|l}
\hline desinenze & verbi & coniugazione \\
\hline -cqui & GIACERE & giacqui, giacesti, giacque, giacemmo, giaceste, giacquero \\
-esti & NASCERE & nacqui, nascesti, nacque, nascemmo, nasceste, nacquero \\
-cque & NUOCERE & nocqui, n(u)ocesti, nocque, $\mathrm{n}(\mathrm{u})$ ocemmo, n(u)oceste, nocquero \\
-emmo & pIACERE & piacqui, piacesti, piacque, piacemmo, piaceste, piacquero \\
-este & tacqui, tacesti, tacque, tacemmo, taceste, tacquero \\
-cquero & &
\end{tabular}

e) Verbi con le forme latine (tu, noi, voi): ponere, traere, -ducere, bevere, dicere, facere

\begin{tabular}{c|c|c|c|c|c}
\hline PORRE & TRARRE & PRODURRE & BERE & DIRE & FARE \\
\hline posi & trassi & produssi & bevvi & dissi & feci \\
\hline ponesti & traesti & producesti & bevesti & dicesti & facesti \\
\hline pose & trasse & produsse & bevve & disse & fece \\
\hline ponemmo & traemmo & producemmo & bevemmo & dicemmo & facemmo \\
\hline poneste & traeste & produceste & beveste & diceste & faceste \\
\hline posero & trassero & produssero & bevvero & dissero & fecero \\
\hline
\end{tabular}

\section{CONCLUSIONI}

In un corso per stranieri, i manuali di lingua - oggi redatti prevalentemente in conformità ai presupposti di un approccio comunicativo (a questo proposito si veda p. es. Balboni 2015), per cui meno attenti alle questioni grammaticali, se non nella loro dimensione pratica o comunicativa, per l'appunto - vengono di solito accompagnati da un testo di grammatica ${ }^{26}$. In effetti, le case editrici specializzate nell'insegnamento dell'italiano L2/LS, accanto ai manuali, hanno nella loro offerta editoriale diversi volumi dedicati interamente alla grammatica che dovrebbero essere - come leggiamo nell' introduzione a uno dei titoli esaminati - uno strumento utile per coloro che "desiderano consolidare e/o approfondire le proprie conoscenze di grammatica italiana" (GT1: 6) sia come studenti nelle scuole e negli istituti italiani sia come "privati residenti in Italia o all'estero", quindi autodidatti (ibid.). Lo studente si aspetta, quindi, da una grammatica - come credo - una presentazione sintetica, approfondita e ordinata che gli permetta di amplificare e completare le sue competenze linguistiche, ma che lo guidi altresì nel suo percorso glottodidattico e che soprattutto faciliti l'apprendimento della lingua italiana.

${ }^{26}$ Sarebbe interessante esaminare anche le sezioni dedicate alla grammatica nei corsi di lingua dal punto di vista delle irregolarità flessive; per le analisi della grammatica nei nuovi manuali di lingua si vedano p. es. Kaliska \& Kostecka-Szewc (2018), Serena (2018), Słapek (2017). 
Quanto alla presentazione delle forme verbali - come abbiamo visto -, sono invece poche le grammatiche della lingua italiana per stranieri in cui si cerca di trovare diverse regolarità o corrispondenze tra i verbi irregolari, il che potrebbe, senza alcun dubbio, facilitare l'apprendimento di tali forme flesse. Gli autori preferiscono invece di solito proporre una semplice lista di verbi irregolari da imparare a memoria.

Tuttavia, benché le loro liste dei verbi irregolari siano abbastanza numerose (ricordiamo gli elenchi più ricchi esposti nei volumi UD1 e UD2: 72 forme di PPass e 46 forme di PR), ci sono alcune inesattezze che andrebbero preferibilmente evitate, quali 1) la presenza dei verbi regolari all'interno dei paragrafi dedicati alla flessione irregolare, come nel caso del PPass: conoscere, stare (2 volumi), ottenere $(1)$, piacere $(5) ; 2$ ) la presenza - in uno stesso volume - di numerosi verbi prefissati che si coniugano come il verbo base; ciò si nota soprattutto nel caso del PPass (p. es. porre, comporre, disporre, esporre, imporre in $\mathrm{GeP}$ ), meno quanto al PR (p. es. porre, opporsi in GB); e - prima di tutto - 3) la mancanza di forme flesse effettivamente irregolari, che non si possono dedurre da altre forme o che non si possono inserire in un gruppo di verbi che dividono certi tratti strutturali; in tal modo, a) per quanto riguarda l'IMP, rimangono dimenticati i verbi: trarre (esposto in 5 volumi), porre (7), nonché i verbi composti con -durre (9); b) per quanto riguarda il PPass, non sono stati presentati da nessuna grammatica i verbi prediligere, scuotere, valere, sono rari anche: distinguere (esposto in un solo volume), fondere (in un volume appare il prefissato confondere), discutere (3), stringere (insieme alla variante costringere appare 2 volte), trarre (insieme a attrarre: 2), volgere (insieme ai prefissati: 3 ), cuocere (6); c) quanto alle forme del PR, rimangono particolarmente trascurati i verbi: stringere (presentato in un solo volume), piovere (3 volumi), parere (4); si confrontino le tabelle 4, 5 e 6 , esposte nell'appendice.

L'aspetto fondamentale, però, per la presentazione delle forme flesse irregolari, cioè quello di far notare agli apprendenti che ci sono numerose corrispondenze formali che potrebbero facilitare lo studio (per tutti e tre $\mathrm{i}$ tempi verbali di cui si è parlato nelle precedenti pagine), è stato - purtroppo - particolarmente trascurato. Confido che questa analisi delle grammatiche, insieme alle proposte della presentazione delle forme flesse irregolari, potrà effettivamente essere d'aiuto per gli autori delle grammatiche italiane per stranieri. 


\section{BIBLIOGRAFIA}

\section{Grammatiche della lingua italiana per stranieri}

Celi, M. \& La Cifra, L. (2011). Grammatica d'uso della lingua italiana. Teoria ed esercizi (A1/B2). Milano: Hoepli.

Chiuchiù, A. \& Chiuchiù, G. (2015). Comunicare in italiano. Grammatica per stranieri con esercizi e soluzioni (A1/C1). Milano: Hoepli.

Colombo, F. (2006). Grammatica e pratica della lingua italiana per studenti stranieri (A2-B1). Recanati: Eli.

Debetto, G. (2016). La lingua italiana e le sue regole (A1-B2). Torino: Loescher.

Duso, E.M. (2019). Grammatica dell'italiano L2 (A1-C2). Roma: Carocci. Ercolino, E. \& Pellegrino, T. A. (2011). L'utile e il dilettevole 1. Esercizi e regole per comunicare (A1/B1). Torino: Loescher.

Ercolino, E. \& Pellegrino T. A. (2012). L'utile e il dilettevole 2. Esercizi e regole per comunicare (B2-C2). Torino: Loescher.

Esposito, A. \& Errico, R. (2007). Grammatica di base. Risorse di grammatica italiana per stranieri (A1/B2). Perugia: Guerra.

Gatti, F. \& Peyronel, S. (2006). Grammatica in contesto. Strutture e temi di italiano per stranieri (A1/B1). Torino: Loescher.

Iacovoni, G., Fiorentino, B. \& Persiani, N. (2009). Gramm.it. Grammatica italiana per stranieri con esercizi e testi autentici (A1/C1). Torino: Bonacci.

Landriani, M. R. (2012). Grammatica attiva. Italiano per stranieri (A1/ B2+). Firenze: Le Monnier.

Latino, A. \& Muscolino, M. (2014a). Una grammatica italiana per tutti 1. Regole d'uso, esercizi e chiavi per studenti stranieri (volume primo: livello elementare; A1/A2). Roma: Edilingua.

Latino, A. \& Muscolino, M. (2014b). Una grammatica italiana per tutti 2. Regole d'uso, esercizi e chiavi per studenti stranieri (volume secondo: livello intermedio; B1-B2; 2a ed.). Roma: Edilingua.

Mezzadri, M. (2003). Italiano essenziale. Testo di grammatica per studenti stranieri dal livello principianti (A1) al livello intermedio-alto (B2). Perugia: Guerra.

Mezzadri, M. (2016). Grammatica pratica della lingua italiana (A1/C1). Torino: Bonacci.

Nocchi, S. (2011). Nuova grammatica pratica della lingua italiana. Esercizi - test-giochi (A1/B2; edizione aggiornata). Firenze: Alma.

Nocchi, S. \& Tartaglione, R. (2006). Grammatica avanzata della lingua italiana (B1-C1). Firenze: Alma. 
Petri, A., Laneri, M. \& Bernardoni, A. (2015). Grammatica di base dell'italiano. La prima grammatica cognitiva dell'italiano (A1-B1). Barcellona: Casa delle Lingue.

Ricci, M. (2011). Via della grammatica. Teoria, esercizi, test e materiale autentico per stranieri (A1/B2, elementare - intermedio). Roma: Edilingua.

Tartaglione, R. \& Benincasa, A. (2015a). Grammatica della lingua italiana Per Stranieri. Regole · esercizi · letture · test (Vol. 1: di base; A1/A2). Firenze: Alma.

Tartaglione, R. \& Benincasa, A. (2015b). Grammatica della lingua italiana Per Stranieri. Regole · esercizi · letture · test (Vol. 2: intermedio-avanzato; B1/B2). Firenze: Alma.

Troncarelli, D. \& La Grassa, M. (2017). GrammaticAvanzata. Esprimersi con le frasi: funzioni, forme e attività (B2+/C2). Roma: Edilingua.

\section{Studi}

Balboni, P. (2014). Didattica dell'Italiano come lingua seconda e straniera. Torino: Loescher/Bonacci.

Balboni, P. (2015). La comunicazione interculturale e l'approccio comunicativo. EL.LE, 4/1, 1-20.

Berretta, M. (1993). Morfologia. In A. A. Sobrero (a cura di), Introduzione all'italiano contemporaneo. Le strutture (pp. 193-245). Bari-Roma: Laterza.

Bertinetto, P. M. (2001). Il verbo. In L. Renzi, G. Salvi, \& A. Cardinaletti (a cura di), Grande grammatica italiana di consultazione, Vol. II. I sintagmi verbale, aggettivale, avverbiale. La subordinazione (nuova edizione, pp. 13-161). Bologna: il Mulino.

Bertinetto, P. M., Burani, C., Laudanna, A., Marconi, L., Ratti, D., Rolando, C., \& Thornton, A.M. (2005). Corpus e Lessico di Frequenza dell'Italiano Scritto (CoLFIS). Disponibile sul sito http://linguistica. sns.it/CoLFIS/Home.htm.

Bertinetto, P. M. \& Squartini, M. (1996). La distribuzione del Perfetto Semplice e Composto nelle diverse varietà di italiano. Romance Philology, 49, 383-419.

Cesarini, S. (2007). Aspetti linguistici. In A. Benucci (a cura di), Sillabo di italiano per stranieri (pp. 127-187). Perugia: Guerra.

D'Achille, P. (2003). L'italiano contemporaneo. Bologna: il Mulino.

Della Valle, V., \& Patota, G. (2015). Viva la grammatica (nuova edizione aggiornata). Milano: Sperling \& Kupfer.

Kaliska, M. \& Kostecka-Szewc, A. (2018). La grammatica all'età adolescente. Soluzioni didattiche applicate in una nuova serie di manuali $\mathrm{Va}$ bene! Italica Wratislaviensia, 9/2, 119-141. 
Palermo, M. (2015). Linguistica italiana. Bologna: il Mulino.

Patota, G. (1987). «Diedi» o «detti». Studi linguistici italiani, 13/1, 102-107. Schwarze, C. (2009). Grammatica della lingua italiana. Roma: Carocci. Sensini, M. (2011). La grammatica della lingua italiana. Milano: Mondadori.

Serena, E. (2018). Modelli di descrizione grammaticale nei manuali di italiano per apprendenti di madrelingua tedesca. Italica Wratislaviensia, 9/1, 247-269.

Słapek, D. (2016). Forme verbali alternative (regolari e irregolari) del passato remoto. Studia universitatis ereditati, 4/1, 85-96.

Słapek, D. (2017). Argomenti grammaticali nei manuali e nei certificati d'italiano LS. Rassegna Italiana di Linguistica Applicata, XLIX, 1/2017, 109-127.

Słapek, D. (2020). Doppia coniugazione regolare del Passato Remoto in italiano contemporaneo: la (falsa?) alternanza tra le forme verbali uscenti in -ei, -é, -erono ed -etti, -ette, -ettero. Zeitschrift für romanische Philologie, 136/1, 246-262.

Squartini, M. (2010). Il verbo. In G. Salvi \& L. Renzi (a cura di), Grammatica dell'italiano antico, Vol. 1 (pp. 511-545). Bologna: il Mulino.

Squartini, M. (2015). Il verbo. Roma: Carocci.

Stoppelli, M. (2004). Italiano. Verbi. Novara: Garzanti Linguistica.

Tekavčić, P. (1980). Grammatica storica dell'italiano. Volume II: Morfosintassi. Bologna: il Mulino.

\section{APPENDICE}

Tabella 4. Verbi irregolari all'imperfetto indicativo nelle grammatiche analizzate

\begin{tabular}{|c|c|c|c|c|c|c|c|c|c|c|c|c|c|c|c|c|c|}
\hline & $\mathrm{CI}$ & GAt & GB & GBI & $\mathrm{GdU}$ & GeP & $\mathrm{GiC}$ & G.it & GL2 & GP & GS1 & GT1 & IE & LSR & NGP & UD1 & VG \\
\hline ESSERE & + & + & + & + & + & + & & + & + & + & + & + & + & + & + & + & + \\
\hline BERE & + & + & + & + & + & + & & + & + & + & + & + & + & & + & + & + \\
\hline DIRE & + & + & + & + & + & + & & + & + & + & + & + & + & & + & + & + \\
\hline FARE & + & + & + & + & + & + & & + & + & + & + & + & + & & + & + & + \\
\hline PORRE & + & & ** & & & * & & & & + & & & + & & & * & $* * *$ \\
\hline TRADURRE & + & & $*$ & & + & + & & & & + & & + & + & & & + & + \\
\hline \multirow[t]{2}{*}{ TRARRE } & & & + & & & + & & & & + & & & + & & & + & \\
\hline & \multicolumn{17}{|c|}{ * prefissati: pro-; ** prefissato: es-; *** prefissato op- } \\
\hline
\end{tabular}


Tabella 5. Verbi irregolari al passato prossimo nelle grammatiche analizzate

\begin{tabular}{|c|c|c|c|c|c|c|c|c|c|c|c|c|c|c|c|c|}
\hline & $\mathrm{CI}$ & GAt & GB & GdU & $\mathrm{GeP}$ & $\mathrm{GiC}$ & G.it & GL2 & GP & GS1 & GT1 & IE & LSR & NGP & UD1 & VG \\
\hline ACCENDERE & & + & + & + & + & + & + & + & + & & + & + & & + & + & + \\
\hline ACCORGERSI & & & & & + & & & & & & & & & + & + & \\
\hline AGGIUNGERE & & + & & & + & & & & & & & & & & + & \\
\hline APRIRE & + & + & + & + & + & + & + & + & + & + & + & + & + & + & + & + \\
\hline ASSISTERE & + & & & & & & & & & & & & & & & \\
\hline ASSUMERE & & & & & + & & & & & & & & & & & \\
\hline ATTENDERE & & + & & & + & & & & & & & & & & + & \\
\hline ATTRARRE & & & & & + & & & & & & & & & & & \\
\hline BERE & + & + & + & + & + & + & + & + & + & + & + & + & + & + & + & + \\
\hline CHIEDERE & + & + & + & + & + & + & + & + & + & + & + & + & + & + & + & + \\
\hline CHIUDERE & + & + & + & + & . & + & + & + & + & + & + & + & + & + & + & + \\
\hline COGLIERE & & & & & & & & & & & & & & & + & \\
\hline COINVOLGERE & & & & & + & & & & & & & & & & & \\
\hline COMPORRE & & & & & + & & & & & & & & & & + & \\
\hline COMPRENDERE & & & & & + & & & & & & & & & & & \\
\hline CONCEDERE & & & & & + & & & & & & & & & & & \\
\hline CONCLUDERE & & & & & & & & & & & & & & & + & \\
\hline CONDIVIDERE & & & & & + & & & & & & & & & & & \\
\hline CONFONDERE & & & & & + & & & & & & & & & & & \\
\hline CONNETTERSI & & + & & & & & & & & & & & & & & \\
\hline CONOSCERE & & & & + & + & & & & & & & & & & & \\
\hline CONTRAPPORRE & & & & & + & & & & & & & & & & & \\
\hline COPRIRE & + & & & & & & & & & & & & + & & + & \\
\hline CORREGGERE & & + & & + & + & & & + & + & & + & + & & & + & \\
\hline CORRERE & + & + & + & & & & + & + & + & & & + & & + & + & + \\
\hline COSTRINGERE & & & & & + & & & & & & & & & & & \\
\hline CUOCERE & & + & & & & & & + & + & & & + & & & + & + \\
\hline DECIDERE & + & + & & & + & + & + & + & + & + & + & + & & + & + & + \\
\hline DESCRIVERE & & + & & & & & & & & & & & & & & \\
\hline DFENDERE & & & & & & & & & & & & & & & + & \\
\hline DIPENDERE & & & & & + & & & & & & & & & & & \\
\hline DIPINGERE & + & & & & + & & & + & & & + & & & & + & + \\
\hline DIRE & + & + & + & + & + & + & + & + & + & + & + & + & + & + & + & + \\
\hline DISCUTERE & & & & & + & & & & & & + & & & & + & \\
\hline DISPORRE & & & & & + & & & & & & & & & & & \\
\hline DISTINGUERE & & & & & + & & & & & & & & & & & \\
\hline
\end{tabular}




\begin{tabular}{|c|c|c|c|c|c|c|c|c|c|c|c|c|c|c|c|c|}
\hline & $\mathrm{CI}$ & GAt & GB & GdU & $\mathrm{GeP}$ & $\mathrm{GiC}$ & G.it & GL2 & GP & GS1 & GT1 & IE & LSR & NGP & UD1 & VG \\
\hline DISTRUGGERE & & & & & & & & & & & & & & & + & \\
\hline DIVIDERE & & & & & + & & & + & + & & & + & & + & + & + \\
\hline EMERGERE & & & & & + & & & & & & & & & & & \\
\hline ESISTERE & & & & & + & & & & & & & & & & & \\
\hline ESPLODERE & & & & & + & & & & & & & & & & & \\
\hline ESPORRE & & & & & + & & & & & & & & & & & \\
\hline ESPRIMERE & + & + & & & + & & & & & & & & & & + & \\
\hline ESSERE & . & + & + & & + & + & + & & + & + & + & + & & + & + & + \\
\hline FARE & + & + & + & + & + & + & + & + & + & + & + & + & + & + & + & + \\
\hline FRIGGERE & & + & & & & & & & & & & & & & + & \\
\hline GIUNGERE & & & + & & & & & & & & & & & & + & \\
\hline IMPORRE & & & & & + & & & & & & & & & & & \\
\hline INCLUDERE & & & & & + & & & & & & & & & & & \\
\hline LEGGERE & + & + & + & + & + & + & + & + & + & + & + & + & + & + & + & + \\
\hline METTERE & + & + & + & + & + & + & + & + & + & + & + & + & + & + & + & + \\
\hline MORDERE & & & & & & & & & & & & & & & + & \\
\hline MORIRE & & + & + & & + & & + & + & + & + & + & + & + & + & + & + \\
\hline MUOVERE & + & + & & & + & & . & 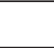 & + & . & & + & & & + & \\
\hline NASCERE & & + & + & & + & & + & + & + & + & & + & + & + & + & + \\
\hline NASCONDERE & & + & & & + & & + & + & + & & & + & & & + & + \\
\hline OFFENDERE & & & & & & & & & & & & & & + & + & + \\
\hline OFFRIRE & + & + & + & + & + & & + & & + & & + & + & + & + & + & + \\
\hline OTTENERE & & & & & + & & & & & & & & & & & \\
\hline PERCORRERE & & & & & + & & & & & & & & & & & \\
\hline PERDERE & + & + & $*$ & + & + & $*$ & + & & + & & + & $*$ & + & + & + & + \\
\hline PERMETTERE & & & & & & & & & & & + & & & & + & \\
\hline PIACERE & & & & & + & & & + & + & & & + & & & & + \\
\hline PIANGERE & & + & & + & + & & + & + & + & & + & + & & & + & + \\
\hline PORGERE & & & & & & & & & & & & & & & + & \\
\hline PORRE & + & & & & + & & & & + & & & + & & & + & \\
\hline PRENDERE & + & + & + & + & + & + & + & + & + & + & + & + & + & + & + & + \\
\hline PRETENDERE & & & & & + & & & & & & & & & & & \\
\hline PREVEDERE & & & & & + & & & & & & & & & & & \\
\hline PROMETTERE & & + & & & & & & & & & + & & & & & \\
\hline PROMUOVERE & & & & & + & & & & & & & & & & & \\
\hline PROPORRE & & + & & & & & & & & & + & & & & + & \\
\hline PROTEGGERE & & & & & + & & & & & & & & & & & \\
\hline PUNGERE & & & & & & & & & & & & & & & + & \\
\hline RACCOGLIERE & & + & & & & & + & & & & & & & & & \\
\hline
\end{tabular}




\begin{tabular}{|c|c|c|c|c|c|c|c|c|c|c|c|c|c|c|c|c|}
\hline & $\mathrm{CI}$ & GAt & GB & GdU & $\mathrm{GeP}$ & $\mathrm{GiC}$ & G.it & GL2 & GP & GS1 & GT1 & IE & LSR & NGP & UD1 & VG \\
\hline RAGGIUNGERE & & & & & + & & & & & & & & & & & \\
\hline RENDERE & + & & + & & + & + & & & & & & & & & + & \\
\hline RICHIEDERE & & & & & + & & & & & & & & & & & \\
\hline RIDERE & & + & & + & & & & & + & & & + & & & & + \\
\hline RIDURRE & & & & & & & & & & & + & & & & & \\
\hline RIMANERE & & + & & & + & + & + & + & + & & + & + & & + & + & + \\
\hline RIMPIANGERE & & & & & + & & & & & & & & & & & \\
\hline RISOLVERE & + & & & & + & & & & + & & & + & & & + & + \\
\hline RISPONDERE & + & + & + & + & + & + & + & + & + & + & + & + & + & + & + & + \\
\hline ROMPERE & + & + & & + & + & + & + & + & + & & + & + & & + & + & \\
\hline SCEGLIERE & + & + & + & + & + & & + & + & + & & + & + & & + & + & + \\
\hline SCENDERE & & & + & & + & + & & + & & & + & & & + & + & \\
\hline SCIOGLIERE & & & & & + & & & & & & & & & & + & \\
\hline SCOMPARIRE & & & & & + & & & & & & & & & & & \\
\hline SCOPRIRE & + & + & & & + & & + & & & + & & & + & & + & \\
\hline SCRIVERE & + & + & + & + & + & + & + & + & + & + & + & + & + & + & + & + \\
\hline SMETTERE & & + & & & & & & & & & & & & & & \\
\hline SOFFRIRE & + & & & & & & & & & & + & & & & + & \\
\hline SORPRENDERE & & & & & & & & & & & & & & & + & \\
\hline SORRIDERE & & + & & & & & & & & & & & & & & \\
\hline SPEGNERE & & + & + & + & + & + & + & + & & & + & & & + & + & + \\
\hline SPENDERE & & + & + & + & + & & + & + & & & + & & + & & + & \\
\hline SPINGERE & & & & & + & & & & & & & & & & + & \\
\hline STARE & & & & & & & & & & & & & & + & & + \\
\hline STRINGERE & & & & & & & & & & & & & & & + & \\
\hline SUCCEDERE & & + & + & & + & + & & & + & & + & + & & + & + & + \\
\hline SVOLGERE & & & & & + & & & & & & & & & & & \\
\hline TENDERE & & & & & + & & & & & & & & & & & \\
\hline TINGERE & & & & & & & & & & & & & & & + & \\
\hline TOGLIERE & & + & & + & & + & & & + & & & + & & & + & + \\
\hline TRADURRE & + & + & & & & $*$ & + & + & + & & + & + & & + & + & + \\
\hline TRARRE & + & & & & & & & & & & & & & & & \\
\hline TRASCORRERE & & + & & & & & & & & & & & & & & \\
\hline UCCIDERE & & & & & + & & & + & + & & & + & & & + & + \\
\hline VEDERE & & + & $* *$ & + & + & $* *$ & + & + & + & + & + & $* *$ & & + & + & + \\
\hline VENIRE & & + & + & & + & + & + & + & + & + & + & + & + & + & + & + \\
\hline VINCERE & & + & + & + & + & + & + & + & + & + & + & + & & + & + & + \\
\hline VIVERE & & + & & & + & + & & + & + & + & + & + & + & + & + & + \\
\hline \multirow[t]{2}{*}{ VOLGERE } & & & & & & & & & & & & & & & + & \\
\hline & \multicolumn{16}{|c|}{${ }^{*}$ perso/perduto $; *$ visto/veduto; in corsivo: verbi regolari } \\
\hline
\end{tabular}


Tabella 6. Verbi irregolari al passato remoto nelle grammatiche analizzate

\begin{tabular}{|c|c|c|c|c|c|c|c|c|c|c|c|c|c|c|c|c|c|}
\hline & $\mathrm{CI}$ & GAt & GB & GBI & $\mathrm{GdU}$ & $\mathrm{GeP}$ & $\mathrm{GiC}$ & G.it & GL2 & GP & GS2 & GT2 & IE & LSR & NGP & UD2 & VG \\
\hline ACCENDERE & & & + & & & & + & & & & & & & & & & \\
\hline ACCORGERSI & & & + & & & & + & & & & & & & & + & & \\
\hline ASSUMERE & + & & & & & & & & & & & & & & & + & \\
\hline ATTENDERE & & + & + & & & & & & & & & & & & + & & \\
\hline AVERE & + & + & + & + & + & + & + & + & + & + & + & + & + & + & + & + & + \\
\hline BERE & + & + & + & $*$ & + & + & + & & + & $*$ & + & + & + & + & + & + & + \\
\hline CADERE & + & + & + & + & & & + & & + & & + & & + & + & + & & \\
\hline CHIEDERE & + & + & + & + & + & & + & + & & + & + & + & + & + & + & + & + \\
\hline CHIUDERE & + & + & + & & + & & + & & & & & & & + & + & + & \\
\hline COGLIERE & & & + & & & & & & & & & & & & & & \\
\hline COMPRENDERE & & & & & & & + & & & & & & & & & & \\
\hline CONCEDERE & + & & & & & & & & & & & & & & & & \\
\hline CONDURRE & & & + & & & & & & & & & & & & & & \\
\hline CONFONDERE & + & & & & & & & & & & & & & & & & \\
\hline CONOSCERE & + & + & + & + & + & & + & & & + & + & + & + & + & + & + & + \\
\hline CORREGGERE & & & & & & & + & & & & & & & & & & \\
\hline CORRERE & + & + & + & + & & & + & & & + & + & + & + & + & + & + & \\
\hline CRESCERE & & & & & + & & & & & & + & & & & & + & \\
\hline CUOCERE & & & & & & & & & & & & & & & & + & \\
\hline DARE & $2 / 1$ & 1 & $1 / 2$ & $1 / 2$ & 1 & $1 / 2$ & $1 / 2$ & 1 & & $1 / 2$ & 1 & $1 / 2$ & $1 / 2$ & 1 & $1 / 2$ & $1 / 2$ & 1 \\
\hline DECIDERE & & + & + & + & & & + & + & & & & + & + & & & + & \\
\hline DIPINGERE & + & & & & & & + & & & & & & & & & + & \\
\hline DIRE & + & + & + & + & + & + & + & + & & & + & + & & & & + & + \\
\hline DIRIGERE & + & & & & & & & & & & & & & & & + & \\
\hline DISCUTERE & & & & & & & & & & & & & & & & + & \\
\hline DISTINGUERE & + & & & & & & & & & & & & & & & + & \\
\hline DISTRARRE & & & & & & & & & & & & & & & & + & \\
\hline ESPELLERE & + & & & & & & & & & & & & & & & & \\
\hline ESPRIMERE & & & & & & & & & & & + & & & & & & \\
\hline ESSERE & + & + & + & + & + & + & + & + & + & + & + & + & + & + & + & + & + \\
\hline FARE & + & + & + & + & + & + & + & + & & + & + & + & + & + & + & + & + \\
\hline FONDERE & & & & & & & & & & & & & & & & + & \\
\hline GIUNGERE & & & & & & & & & & & + & & & & & & \\
\hline LEGGERE & + & + & + & & + & + & + & + & & & & & & & + & + & + \\
\hline METTERE & + & + & + & + & + & + & + & + & & + & + & + & + & & + & & + \\
\hline MUOVERE & + & & + & & & & & & & & + & & & & + & + & \\
\hline
\end{tabular}




\begin{tabular}{|c|c|c|c|c|c|c|c|c|c|c|c|c|c|c|c|c|c|}
\hline & $\mathrm{CI}$ & GAt & GB & GBI & GdU & $\mathrm{GeP}$ & $\mathrm{GiC}$ & G.it & GL2 & GP & GS2 & GT2 & IE & LSR & NGP & UD2 & VG \\
\hline NASCERE & + & + & + & + & + & & + & + & & + & + & & + & & + & + & + \\
\hline NASCONDERE & & & & & & & + & & & & + & & & & & & \\
\hline OPPORSI & & & + & & & & & & & & & & & & & & \\
\hline PARERE & & + & + & & & & & & & & & & & & + & + & \\
\hline PERDERE & + & + & + & + & + & & + & & & + & & & + & & + & + & \\
\hline PIACERE & + & & + & & & & + & & + & & + & & & + & + & & \\
\hline PIANGERE & & & & & & & + & & + & & & & & & & + & \\
\hline PIOVERE & & & & & & & + & & + & & + & & & & & & \\
\hline PRENDERE & + & + & + & + & + & + & + & + & & + & & + & + & & + & + & \\
\hline PORRE & + & & & & & & & & & & + & & & & & & \\
\hline PRODURRE & & & & & & & + & & & & & & & & & + & \\
\hline PROPORRE & & & & & & + & & & & & & & & & & & \\
\hline RACCOGLIERE & + & & & & & & & & & & & & & & & & \\
\hline REGGERE & & & & & & & & & + & & & & & & & & \\
\hline RENDERE & & & & & & & + & & & & & & + & & & & \\
\hline RIDERE & + & & + & & & & + & & + & & & & & & & & \\
\hline RIMANERE & + & + & + & & & + & + & & & & + & & & + & + & + & + \\
\hline RISOLVERE & + & & & & & & & & & & + & & & & & & \\
\hline RISPONDERE & + & + & + & + & + & & + & + & & & + & + & + & + & + & + & \\
\hline ROMPERE & + & + & + & + & & & + & & & + & + & & + & & + & + & \\
\hline SAPERE & + & + & + & + & + & + & + & & + & + & + & + & + & + & + & + & + \\
\hline SCEGLIERE & & & + & & & & + & & + & & & & & & + & & \\
\hline SCENDERE & & & + & & & & + & & & & & + & & & + & + & \\
\hline SCIOGLIERE & & & & & & & & & & & & & & & & + & \\
\hline SCOMPARIRE & & & & & & & & & & & & & & & & + & \\
\hline SCRIVERE & + & + & + & + & + & & + & + & & + & + & & + & & + & + & + \\
\hline SPEGNERE & & & & + & & & & & + & + & + & & + & & + & & + \\
\hline SPENDERE & & + & + & & & & + & & + & & + & & & & & & \\
\hline STARE & + & + & + & + & & + & + & & & + & + & + & + & + & + & + & + \\
\hline STRINGERE & & & & & & & & & & & + & & & & & & \\
\hline SUCCEDERE & & & & & & & & & & & + & & & & & & \\
\hline TACERE & + & & + & & & & & & & & & & & & & + & \\
\hline TENERE & + & + & + & + & & & + & & + & + & + & + & + & + & & + & \\
\hline TOGLIERE & & & & & & & + & & & & & & & & & + & \\
\hline TRADURRE & + & & + & & & + & + & & & & + & & & & + & + & \\
\hline TRARRE & + & & + & & & + & & & & & + & & & & & & \\
\hline VALERE & & & & & & & & & & & & & & & & + & \\
\hline VEDERE & + & + & + & + & + & & + & + & & + & + & + & + & + & + & + & + \\
\hline VENIRE & + & + & + & + & + & + & + & + & + & + & + & + & + & + & + & + & + \\
\hline
\end{tabular}




\begin{tabular}{l|c|c|c|c|c|c|c|c|c|c|c|c|c|c|c|c|c|c}
\hline & CI & GAt & GB & GBI & GdU & GeP & GiC & G.it & GL2 & GP & GS2 & GT2 & IE & LSR & NGP & UD2 & VG \\
\hline VINCERE & + & & + & & & & + & & + & & + & + & & & & + & \\
\hline VIVERE & + & + & + & & + & & + & + & & + & + & & + & & & + & + \\
\hline VOLERE & + & + & + & + & + & & + & & + & + & + & + & + & + & & & + \\
\hline
\end{tabular}

THE PRESENTATION OF INFLEXIONAL (IR)REGULARITIES IN ITALIAN GRAMMAR TEXTBOOKS FOR FOREIGN LANGUAGE LEARNERS: THE CASE OF THE PAST TENSES OF THE INDICATIVE MOOD

\section{Summary}

The verbal system, without a doubt, is among the most difficult grammatical issues of the Italian language for foreign learners. The root of its difficulty lies in its being characterized, among other things, by a complicated inflexional morphology, not only regarding the number of verbal moods and tenses that the student must learn, but also regarding the abundance of irregular forms that this system contains. In this article, I attempt to examine how various inflexional irregularities are presented in Italian grammar books for foreign language learners; for this purpose, I have analyzed 18 textbooks that make explicit reference to the Common European Framework of Reference. The analysis focuses on the conjugation of three Italian indicative past tenses: the imperfetto, the passato prossimo (I consider the forms of the participio passato, and for this reason other compound past tenses remain outside of my scope of interest in this article), and the passato remoto. In addition to offering a detailed comparative analysis of all the textbooks, each paragraph ends with a didactic proposal for the presentation of these irregular verbal forms.

Keywords: Italian non-mother tongue, teaching grammar, teaching Italian as a foreign language, verbal inflexion, irregular verbs. 